SUPPORTING INFORMATION

\title{
Additive contact polarization of non-ferroelectric polymers for patterning of multilevel memory elements
}

Witold Adamkiewicz ${ }^{1,2}$, Marta M. Siek ${ }^{1 \ddagger}$, Tomasz W. Mazur ${ }^{1}$, Sławomir Lach ${ }^{1}$, Bartosz A. Grzybowski ${ }^{1,2 *}$

${ }^{1}$ Center for Soft and Living Matter, Institute for Basic Science (IBS), Ulsan 44919, Republic of Korea

${ }^{2}$ Institute of Organic Chemistry, Polish Academy of Sciences, Kasprzaka 44, 01-224 Warsaw, Poland

These authors contributed equally to this work *Corresponding author. E-mail: nanogrzybowski@gmail.com (B. A. G.)

\section{Section 1. Materials and methods}

The p-type, B doped silicon substrates (volume electrical resistivity $6.5 \times 10^{-2} \Omega \cdot \mathrm{m}$ ) were purchased diced from MTI Korea, washed with dichloromethane and isopropyl alcohol, and dried in the stream of nitrogen before use. Poly(methyl methacrylate) (PMMA, MW $=350 \mathrm{k}$, Sigma-Aldrich), $0.075 \mathrm{~g} / \mathrm{mL}$, was dissolved by sonication using dry chloroform (Sigma-Aldrich) and filtered through $0.2 \mu \mathrm{m}$ PTFE filter (Millipore). Polyvinylpyrrolidone (PVP, MW $=40 \mathrm{k}$, Sigma-Aldrich), $0.15 \mathrm{~g} / \mathrm{mL}$, was dissolved in absolute ethanol (HPLC grade, Fisher Scientific) by sonication and filtered through $0.2 \mu \mathrm{m}$ PTFE filter. Poly(vinylidene fluoride) (PVDF, MW $=$ 275k, Sigma-Aldrich)/PMMA blend was prepared according to literature protocol. ${ }^{\mathrm{S1}} 0.075 \mathrm{~g} / \mathrm{mL}$ (PVDF/PMMA w/w ratio 4:1) quantities of polymers were dissolved in dimethylformamide (DMF, Sigma-Aldrich) by sonication and filtered through $0.2 \mu \mathrm{m}$ PTFE filter. Poly(vinyl 
acetate) (PVAc, MW $=500 \mathrm{k}$, Sigma-Aldrich), $0.05 \mathrm{~g} / \mathrm{mL}$ was dissolved by sonication in dry chloroform (Sigma-Aldrich) and filtered through $0.2 \mu \mathrm{m}$ PTFE filter. Poly(vinyl chloride) (PVC, high molecular weight, Sigma-Aldrich), $0.05 \mathrm{~g} / \mathrm{mL}$ was dissolved by sonication in dry cyclopentanone (Sigma-Aldrich) and filtered through $0.2 \mu \mathrm{m}$ PTFE filter.

Polymer thin films were spin-coated $\left(125 \mu \mathrm{L} / \mathrm{cm}^{2}, 5000 \mathrm{rpm}, 30 \mathrm{~s}\right)$ in the atmosphere of dry nitrogen $(\mathrm{RH}=19 \%)$. The as-prepared films were sealed in Petri-dishes and dried overnight at $65^{\circ} \mathrm{C} . \mathrm{PVDF} / \mathrm{PMMA}$ blend films were annealed in air according to literature procedure ${ }^{\mathrm{S} 1}$ to achieve low surface roughness. All concentrations were chosen to yield $\sim 1-\mu$ m-thick films after spin-coating.

Photolithography masks (chromium on soda lime glass) with patterns (lines, squares, QR codes) were fabricated by Microimage Korea. Masters were made using SU8-2010 (MicroChem) negative epoxy photoresist on 4" Si wafers (MTI Korea) according to manufacturer's protocol. Masters were hard-baked for $30 \mathrm{~min}$ in $200^{\circ} \mathrm{C}$ to ensure full curing of the photoresist.

Polydimethylsiloxane (Sylgard 184, Dow Corning, lot \#0009004705) was prepared by mixing elastomer base with a crosslinker in a 5:1 w/w ratio (unless stated otherwise). Prepolymer was degassed under vacuum $(5 \mathrm{mbar}, 1 \mathrm{~h})$, cast onto a previously silanized master $(1 \mathrm{H}, 1 \mathrm{H}, 2 \mathrm{H}, 2 \mathrm{H}-$ perfluorooctyltrichlorosilane, Sigma-Aldrich), degassed once more, and cured for $36 \mathrm{~h}$ at $65{ }^{\circ} \mathrm{C}$. After curing, the PDMS mold was gently peeled off the wafer, cut into pieces $(1 \times 1 \times 0.6 \mathrm{~cm})$, thoroughly rinsed with dichloromethane and isopropyl alcohol, and dried under a stream of nitrogen. Prior to contact-charging, PDMS pieces were discharged using soft x-ray photoionization technique ${ }^{\mathrm{S} 2}$ (Hamamatsu PhotoIonBar L12536) for $330 \mathrm{s.}$ 
Metallized PDMS stamps were made using Quorum Technologies Q150RS magnetron sputter coater with $\mathrm{Au} / \mathrm{Pd}(99.99 \%$, Taewon Scientific Co. Ltd.) as a source. The thickness of the layer was set to $100 \mathrm{~nm}$ with a sputtering current of $57 \mathrm{~mA}$.

In a standard stamping protocol, the bare PDMS stamp was placed onto a substratesupported polymer film and left for, typically, 45 min to ensure conformal contact between the two materials. The stamp was then manually peeled off in air. Antistatic tweezers with carbon fiber tip were used for handling the samples (Ideal-tek 249CF.SA). Hand of the person holding the tweezers was grounded using an antistatic wrist strap. All experiments were carried out under ambient conditions (temperature ca. $25{ }^{\circ} \mathrm{C}, \mathrm{RH} \approx 25-45 \%$, the pressure of contact was $\sim 37 \mathrm{~Pa}$ ). The pressure of contact is stated only for informative reasons since we established in our previous work that it does not influence strongly the charges generated during contact electrification. ${ }^{\mathrm{S} 3}$

For micro-contact printing, the metalized PDMS stamp was placed onto a substratesupported PMMA film, and an electrical bias was applied to the stamp for the desired time. The second electrode was placed at the bottom of the substrate.

Kelvin Probe Force Microscopy (KPFM) and Piezoresponse Force Microscopy (PFM) measurements were conducted on a Bruker Dimension Icon Atomic Force Microscope with Peak Force KPFM and PFM modules using PFQNE-Al and SCM-PIT V2 tips. Unless otherwise stated, KPFM imaging was performed with $100 \mathrm{~nm}$ lift height as suggested by Bruker. KPFM potential images were constructed by recording and plotting a map of nulling potential applied to the tip (potential needed to nullify surface potential of the sample) which means the real surface potential is of opposite polarity to the one presented in the images. PFM imaging ( $10 \mathrm{~V}$ bias 
applied to the sample) included only vertical domains. Image acquisition time was 30 min per frame. All AFM images were analyzed using Gwyddion 2.51 software. In order to extract numerical data from KPFM/PFM scans (i.e., height of transferred material, magnitude of potential/phase/amplitude readout), images were analyzed using Gwyddion 2.51 software. We started with background correction by mean-plane subtraction. Next, we used "Extract profiles" tool to draw lines perpendicular to our features in the chosen places. The profiles thus obtained were analyzed using "Critical dimension" tool and their heights were exported for further analysis in Origin Pro 2015.

Scanning Kelvin Probe (SKP) measurements were performed using KP Technologies Ltd. SKP5050 setup equipped with $50 \mu \mathrm{m}$ tips. Data was analyzed using OriginPro 2015 (OriginLabs). FEM simulations were performed using COMSOL Multiphysics 5.1 software package.

Section 2. Further comments on the scanning probe microscopy for the detection of electromechanical signals

Polarization in ferroelectric materials is well documented, both from theoretical as well as experimental points of view. Among polymers, polyvinylidene fluoride (PVDF) and its derivatives, exhibit the ferroelectric type of electric polarization due to a flexible and wellordered structure. The ferroelectricity here arises from the collective and stable ordering of permanent electric dipoles as the electric field is applied. ${ }^{\mathrm{S}}$ However, vast majority of other polarizable polymers do not exhibit ferroelectric polarization but rather metastable electret 
orientation or nonuniformity of space-charge. ${ }^{\mathrm{S} 5}$ Switching/inducing mechanisms in polymeric materials are significantly more complicated than in crystals. They may involve, among others, ion migration, charge trapping, intermolecular charge transfer, etc. ${ }^{\mathrm{S} 6}$ Despite decades of experimental effort, however, the mechanism(s) of polarization in dielectrics is still incompletely understood. ${ }^{\mathrm{S} 7}$

One of the key techniques used to study polarization state of a material is scanning probe microscopy (with special emphasis on Kelvin probe force microscopy, KPFM, and piezoresponse force microscopy, PFM). KPFM has been used for qualitative analysis of polarization in ferroelectric materials for over 20 years. ${ }^{58}$ In this technique, the cantilever is driven above the surface of the sample. The tip is biased with $V_{\text {tip }}=V_{d e}+V_{a c} \cos (\omega t)$, where $V_{a c}$ is the driving voltage. The capacitive force between the tip and the surface potential depends on the tip geometry, surface geometry and topography, as well as tip-surface distance (lift height). The feedback loop is used to nullify the first harmonic of this capacitive force by adjusting the $V_{d e}$ of tip bias so the $V_{d e}$ has magnitude equal to the surface potential. The readout of this technique is a map of the nulling potential. It has to be noted that the geometry of the tip/cantilever as well as that of the surface both influence the readout of the size and potential values of the tested features (both are under-evaluated when the distance between a sample and a tip/cantilever increases). Theoretical calculations prove that it is not possible to extract the absolute value of polarization from the KPFM measurement. ${ }^{\mathrm{S} 8}$ However, if one keeps the parameters of the tip ${ }^{\mathrm{S} 9, \mathrm{~S} 10}$ (i.e., material, shape, and, lift height) and the surface (e.g., viscous damping) constant, it is possible to read the relative values of polarization which are linear with respect to the potential readout of KPFM. It has to be stressed that KPFM measurements do not change the initial polarization of the sample. 
In PFM, the biased tip scans the surface of the sample in contact mode. The inverse piezoelectric effect deforms the sample, and the electromechanical signal is measured as the first harmonic component of the tip deflection, $d=d_{0}+A \cos (\omega t+\varphi)$. The amplitude $A$, assuming ideal contact between the tip and the surface of piezoelectric material, defines the local piezoresponse. This amplitude is determined by the combination of system's geometry, electroelastic properties of the tested material, and of the tip. The phase $\varphi$ of the surface oscillations provides the information about the direction of polarization - when the tip is positively biased, $\varphi=0$ when the polarization is pointing downwards and $\varphi=180^{\circ}$ when the polarization is pointing upwards.

PFM allows for the acquisition of more quantitative data about sample's polarization than KPFM, although the interpretation is even less straightforward. This is due to the origins of PFM signal itself ${ }^{\mathrm{S} 11, \mathrm{~S} 12}$ - it may derive not only from ferroelectric switching but also from migration of ionic species inside the tested material (Vegard strain, like in $\mathrm{SrTiO}_{3}{ }^{\mathrm{S} 13, \mathrm{~S} 14}$ ), formation of dipoles on the surface or in the bulk of the tested material (e.g., in electrets), field effects ${ }^{\mathrm{S} 15, \mathrm{~S} 16}$ (which can be avoided when using a proper tip ${ }^{\mathrm{S17}}$ ), Joule heating and/or electrochemical effects like vacancy ordering, material deposition or permanent damage of the sample. It should also be noted that the contrast mechanism in PFM may be influenced by the applied bias ${ }^{\mathrm{S} 8}\left(V_{a c}\right.$ can modify the initial polarization of the material).

For more thorough theoretical and experimental discussion, the reader is referred to excellent works of M. Cuniot-Ponsard ${ }^{\mathrm{S} 8}$ and S. Kalinin ${ }^{\mathrm{S} 18-\mathrm{S} 20}$ PFM and its uses in the analyses of ferroelectric and non-ferroelectric materials was thoroughly reviewed by, among others, Kalinin et al. ${ }^{\mathrm{S} 21}$, Denning et al. ${ }^{\mathrm{S} 22}$, Seol et $_{\text {al. }}{ }^{\mathrm{S} 12}$ and Vasudevan et al. ${ }^{\mathrm{S} 11}$ 
As discussed in the main text, in our system the main source of the PFM signal is the induced polarization of the electret (for contact-electrified PMMA and PVAc). This assumption is in agreement with previous experiments on electrets. ${ }^{\mathrm{S} 5}$ However, induced polarization is not the only form of electromechanical coupling one should consider for our system. For example, all insulators can exhibit deformations upon application of the electric field ${ }^{\mathrm{S} 23}$ (electrostriction and/or Maxwell stress) which can also be detected with AFM. ${ }^{\text {S24,S25 }}$ But Maxwell stress-induced deformation of the material does not change its direction when the electric field is reversed. ${ }^{\text {S23 }}$ In our case, the phase readout of PFM (which depends on the orientation of polarization) changes direction (i) upon changing the sign of potential applied to metallized stamps (Figure S10), and (ii) when the direction of the applied bias during PFM measurement is reversed (Figure S11). Furthermore, if the electret in our system were not polarized, the PFM image would be the same with or without application of AC bias to the tip. Figure S11 shows this is not the case - the stamped linear features on PMMA are not visible when there is no AC bias applied. If the polarization readout were solely due to mass transfer, the PFM signal should also be visible on stamped bare Si (Figure S3) - in reality, it is not. Altogether, these results exclude the electrostriction/Maxwell stress as the primary/main deformation cause in our electret-based system. 
Table S1 Properties of poly(methyl metacrylate), PMMA, poly(vinyl acetate), PVAc, polyvinylpyrrolidone, PVP, polyvinyl chloride, PVC, polystyrene, PS and poly(4vinylphenol), PVPh.

\begin{tabular}{|c|c|c|c|c|c|c|}
\hline & PMMA & PVAc & PVP & PVC & PS & PVPh \\
\hline $\begin{array}{l}\text { monomer } \\
\text { structure }\end{array}$ & & & $\mathrm{H} \uparrow$ & {$\left[\begin{array}{ll}1 & 1 \\
H & H\end{array}\right]$} & {$\left[\begin{array}{ll}1 & C \\
1 & C \\
H & 1 \\
H & H\end{array}\right]_{n}$} & $\mathrm{OH}_{n}$ \\
\hline $\begin{array}{c}\text { monomer } \\
\text { dipole moment } \\
{[\mathrm{D}]}\end{array}$ & $1.67^{\mathrm{S} 26}$ & $1.75^{\mathrm{S} 27}$ & $3.53^{\mathrm{S} 28}$ & $1.42^{\mathrm{S} 29}$ & $0.13^{\mathrm{S30}}$ & $1.34^{\mathrm{S} 30}$ \\
\hline $\begin{array}{l}\text { Polymer mean } \\
\text { square dipole } \\
\text { moment }\left(\mu^{2} / \mathrm{x}\right) \\
\text { in solution } \\
{\left[\mathrm{D}^{2}\right]^{331}}\end{array}$ & $1.77-2.31$ & 2.89 & $6.70^{\mathrm{S} 32}$ & 1.72 & 0.05 & $1.6^{\mathrm{S} 33}$ \\
\hline $\mathrm{MW}\left[\mathrm{g} \cdot \mathrm{mol}^{-1}\right]$ & $350 \mathrm{k}$ & $500 \mathrm{k}$ & $40 \mathrm{k}$ & $85 \mathrm{k}$ & $280 \mathrm{k}$ & $25 \mathrm{k}$ \\
\hline $\begin{array}{l}\text { density } \\
{\left[\mathrm{g} \cdot \mathrm{cm}^{-3}\right]}\end{array}$ & 1.17 & 1.19 & 1.2 & 1.4 & $0.96-1.04$ & 1.16 \\
\hline $\begin{array}{c}\text { relative } \\
\text { dielectric } \\
\text { constant }^{\mathrm{S} 34}\end{array}$ & 3.0 & 3.2 & $2.67^{\mathrm{S} 35}$ & 3.0 & $2.5^{\mathrm{S} 35}$ & 4.5 \\
\hline $\begin{array}{l}\text { mobility of } \\
\text { charge carriers } \\
{\left[\mathrm{cm}^{2} \cdot \mathrm{V}^{-1} \mathrm{~s}^{-1}\right]^{\mathrm{S34}}}\end{array}$ & $\begin{array}{l}3.5 \times 10^{-9} \\
\text { (Lucite) / } \\
3.6 \times 10^{-11} \\
\text { (Perspex) }\end{array}$ & $2.2 \times 10^{-12}$ & $1 \times 10^{-2}$ & $7 \times 10^{-4}$ & $1.4 \times 10^{-11}$ & $1 \times 10^{-5 \mathrm{~S} 36}$ \\
\hline $\begin{array}{l}\text { volume } \\
\text { resistivity } \\
{[\Omega \cdot \mathrm{cm}]^{\mathrm{S37}}}\end{array}$ & $10^{15}-10^{17}$ & $10^{13}-10^{14}$ & $10^{7}-10^{8 S 38}$ & $6 \times 10^{15}$ & $10^{16}$ & no data \\
\hline $\begin{array}{c}\text { stability of } \\
\text { patterns }\end{array}$ & yes & yes & not tested & no & no patterns & no patterns \\
\hline
\end{tabular}




\section{Section 3. Additional figures}

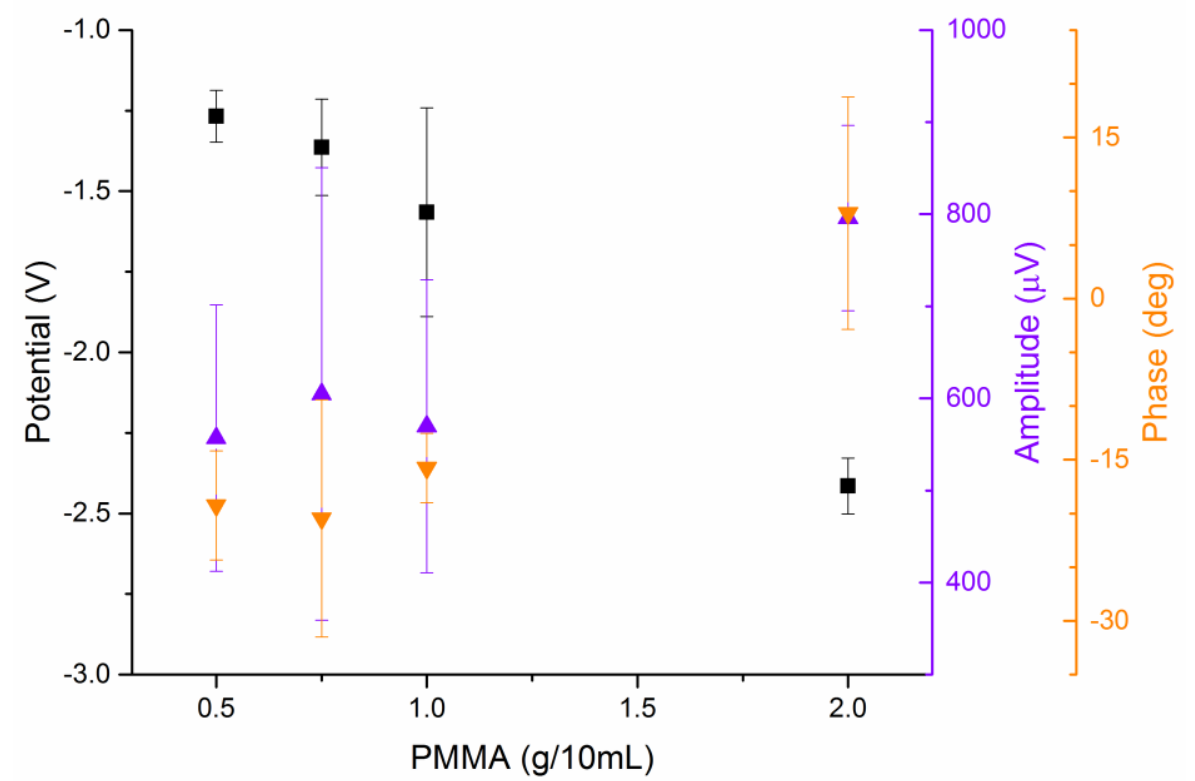

Figure S1. Dependence of the KPFM potential readout and PFM amplitude and phase readout on the thickness of PMMA film spin-coated at 5000 rpm on p-type, B doped Si. X-axis labels denote the concentration of PMMA in $\mathrm{CHCl}_{3}$ used for fabrication of the film (the more concentrated solution, the thicker the resulting film). Data shows mean values \pm SD from 9 profiles. 


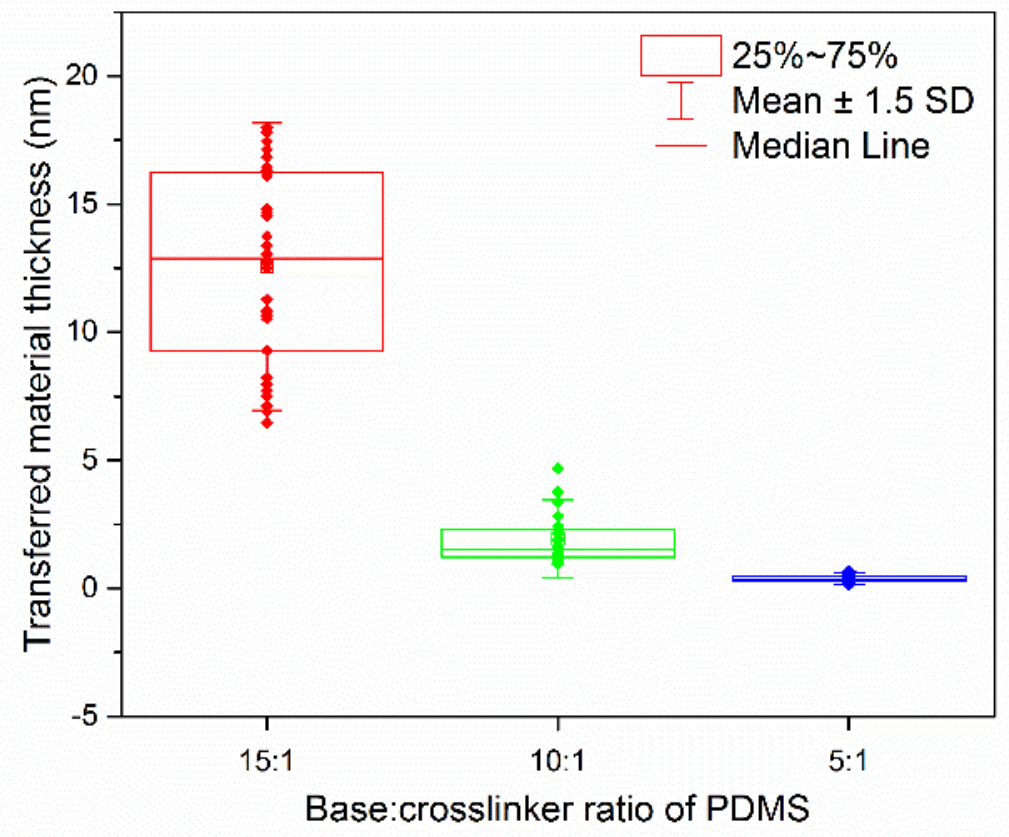

Figure S2. Dependence of the thickness of material transferred after $45 \mathrm{~min}$ of stamp-substrate contact on the base:crosslinker composition of PDMS. Measurements were performed for at least 24 profiles (at least four separate samples) for each type of PDMS. 

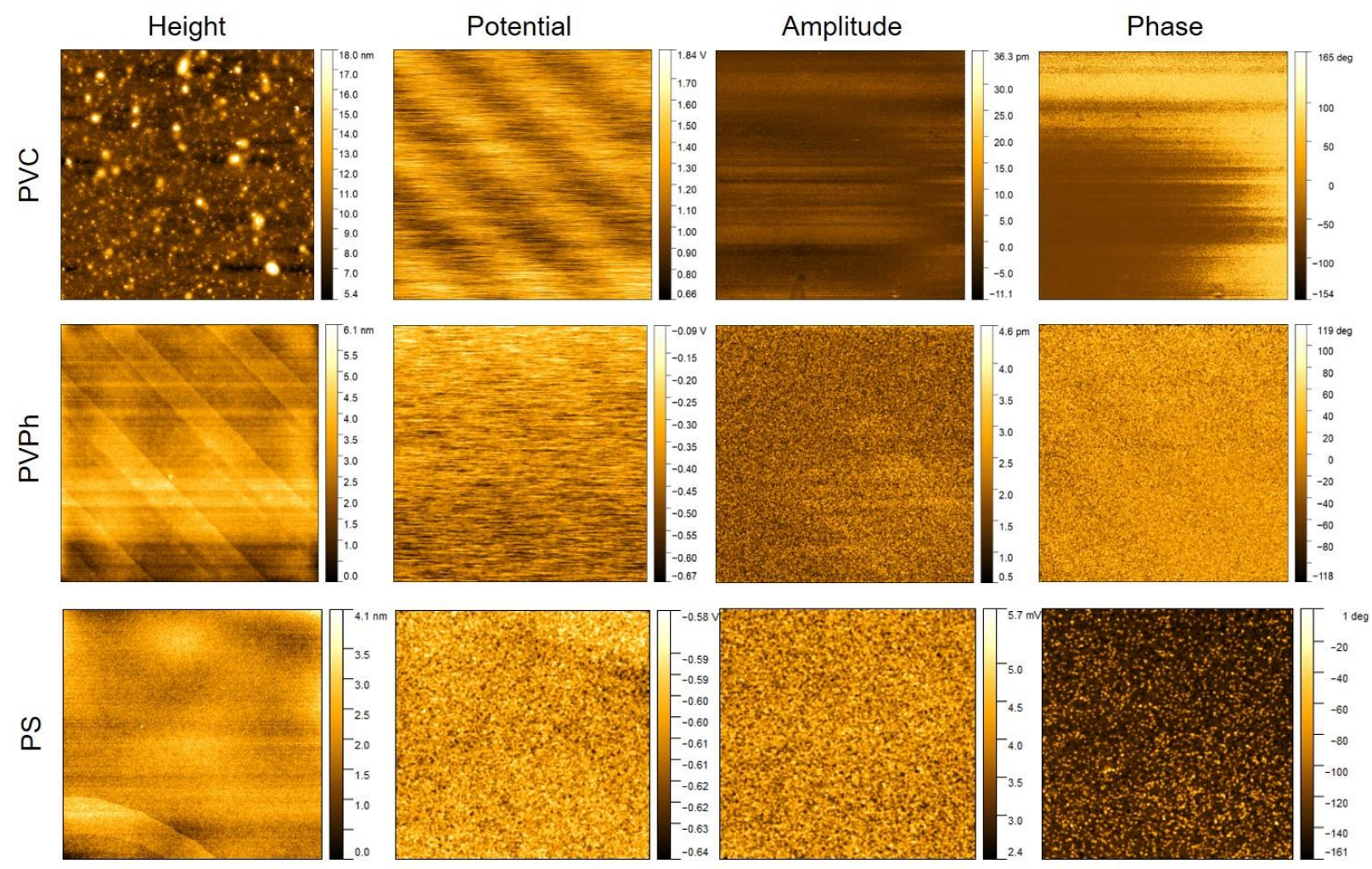

Figure S3. KPFM (height and potential) and PFM (amplitude and phase) signals for PVC, PVPh and PS films spin-coated on p-type B doped Si and contact electrified (after 45 min of stampsubstrate contact) with a 5:1 PDMS stamp presenting an array of parallel-line features. Frame size is $50 \times 50 \mu \mathrm{m}$ for PVC and PVPh and $30 \times 30 \mu \mathrm{m}$ for PS. Images were background corrected by mean-plane subtraction and denoised using 2 px Gaussian filter (for potential, amplitude and phase). 

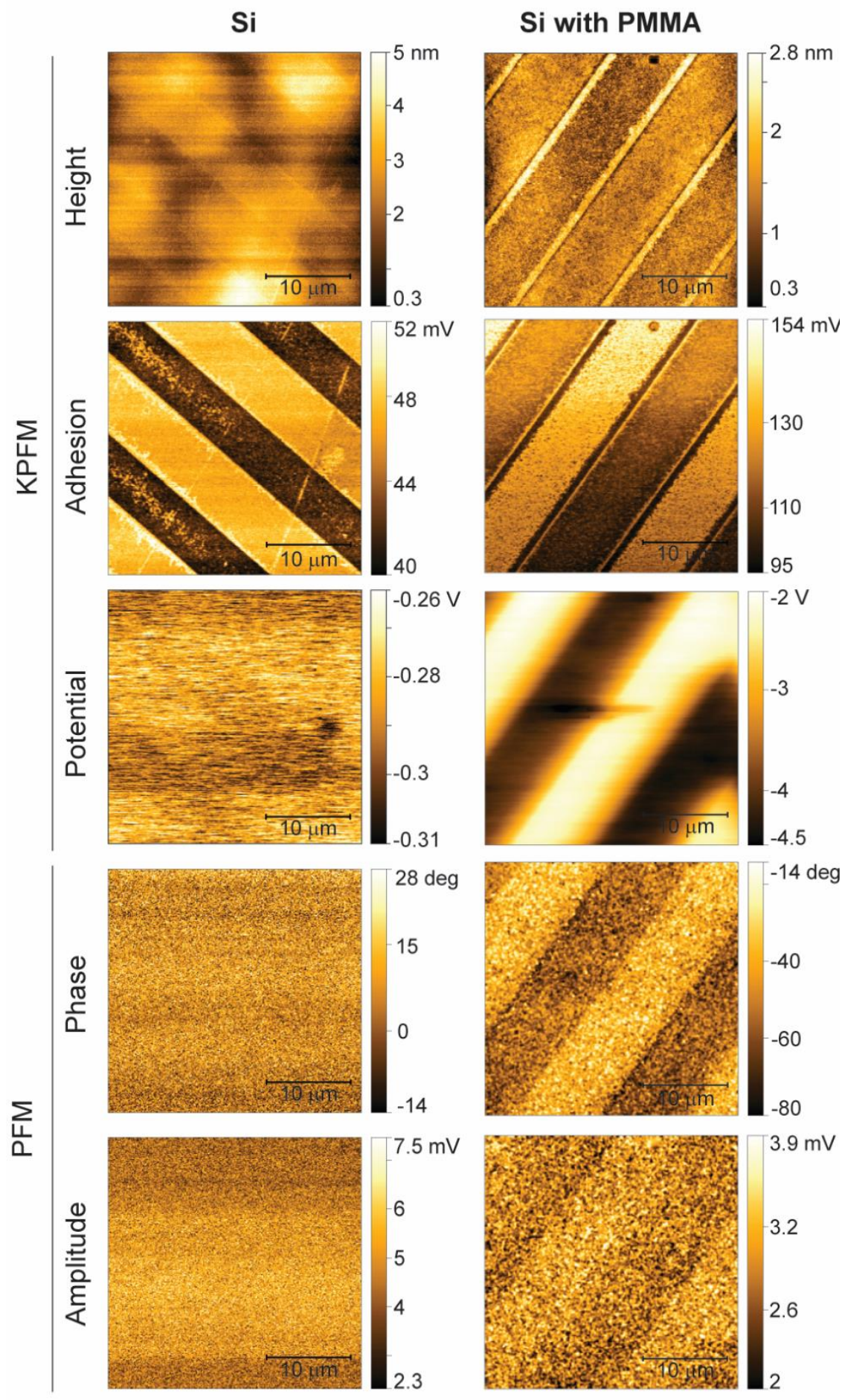

Figure S4. Contact electrification of 5:1 PDMS stamp on bare Si and on Si coated with PMMA (45 min). KPFM images are shown without any data processing. PFM images were denoised using 2 px Gaussian filter. 

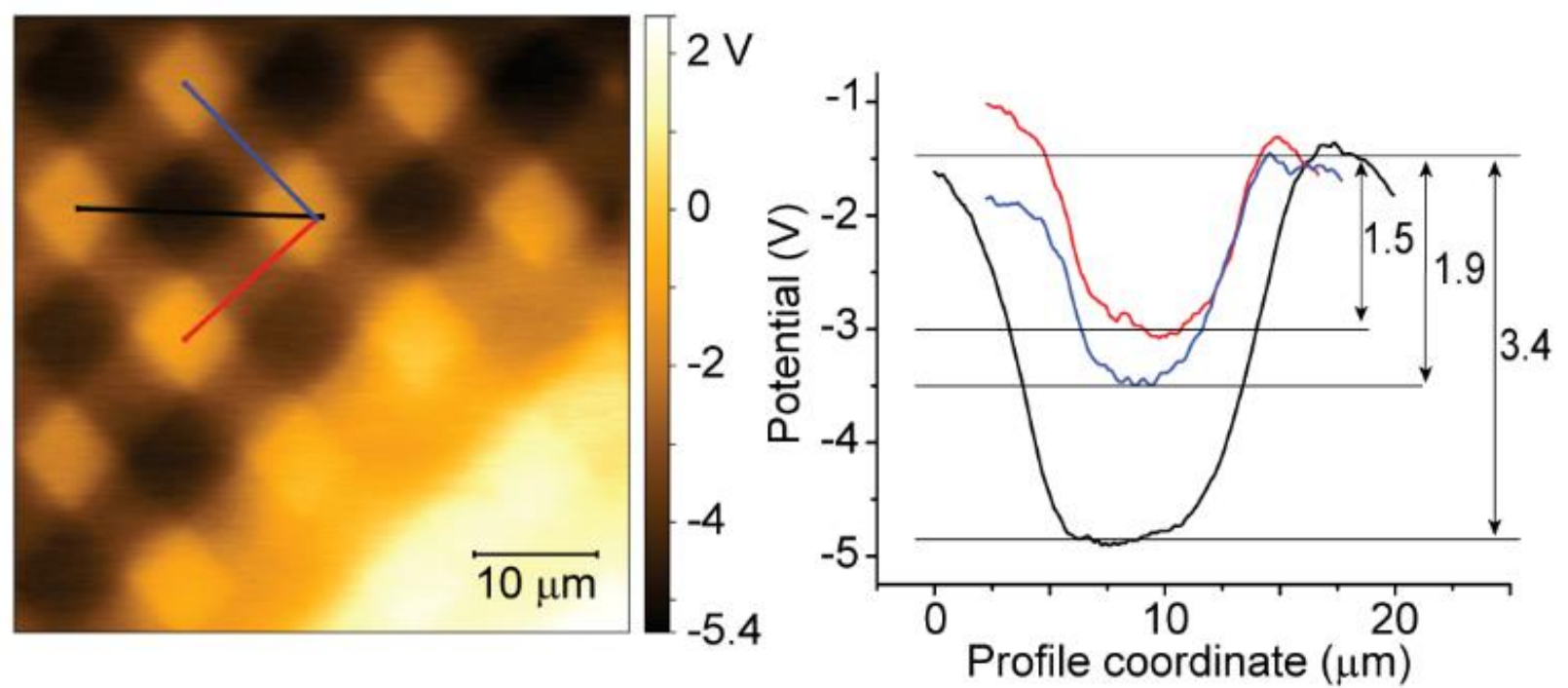

Figure S5. Additivity of KPFM signal for PVAc film supported on p-type, B doped Si. The film was contact-electrified twice with line-featured 5:1 PDMS stamp (after 45 min stamp-substrate contact for each stamping). Right panel plots profiles marked in the left panel (KPFM potential image) with corresponding colors.

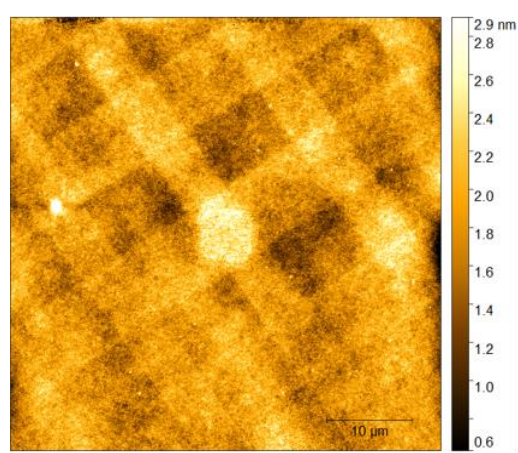

Height

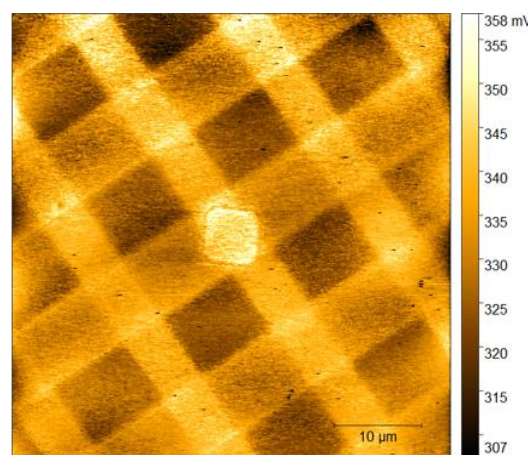

Adhesion

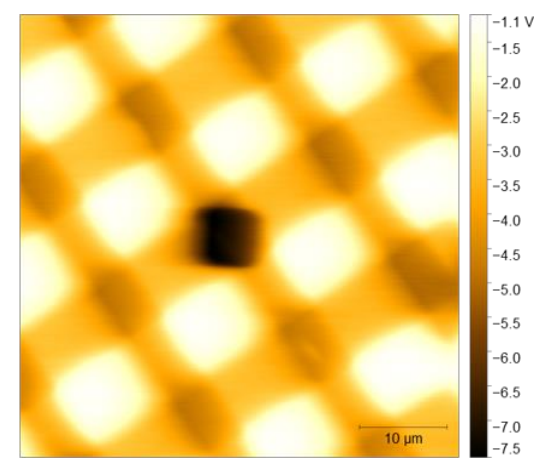

Potential

Figure S6. KPFM images of height, adhesion, and potential of the sample shown in Figure 4e.

For clarity of presentation, height image was background-corrected using Gwyddion software. 

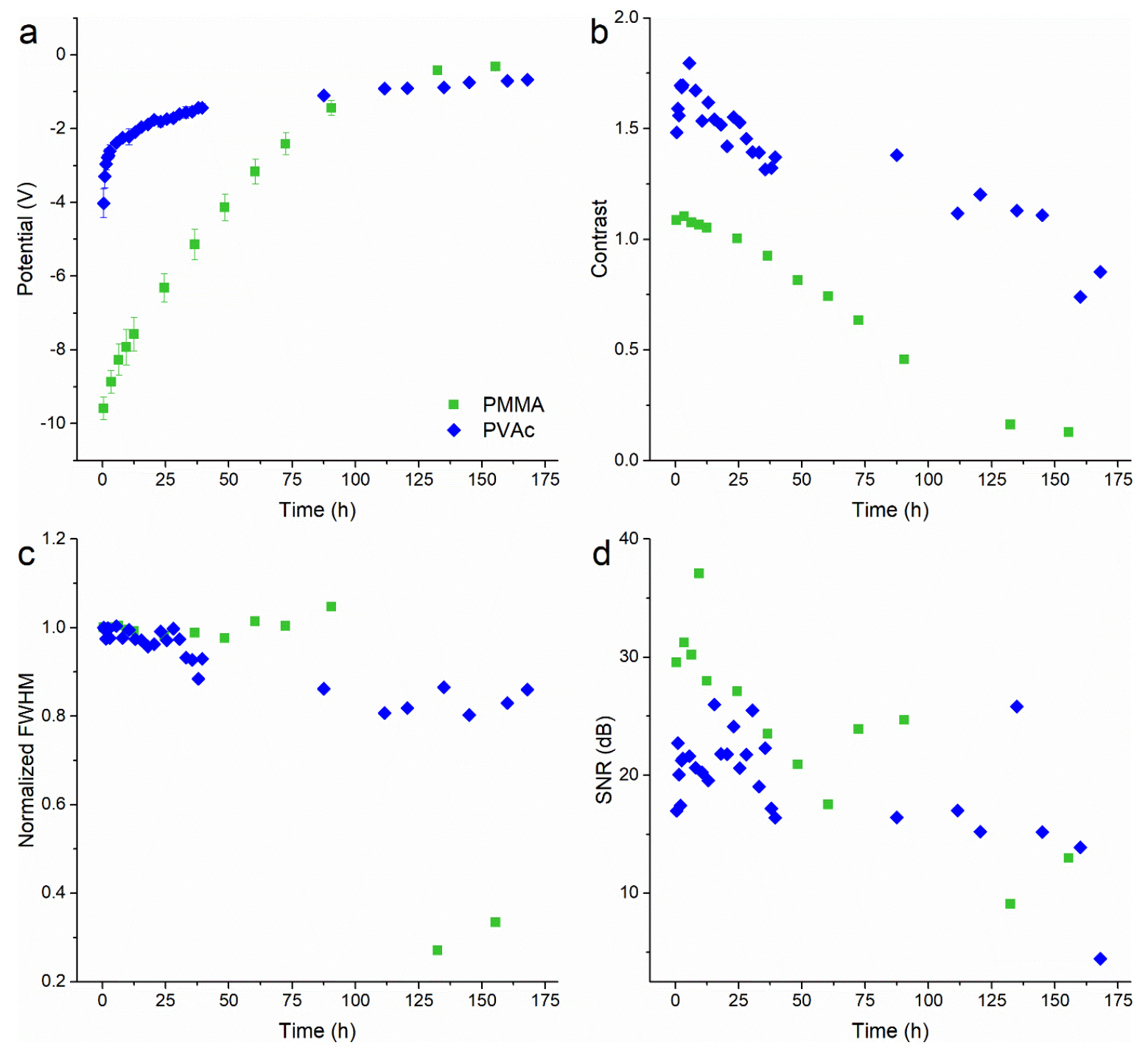

Figure S7. Long-term decay of potential readout after stamping of PMMA (green squares) or PVAc (blue diamonds) films contact electrified with 5:1 PDMS stamps (after 45 min of stampsubstrate contact). Potential (a) is the height of the peak on the KPFM potential readout, contrast (b) is defined as $\left(V_{\max }-V_{\min } / V_{\max }+V_{\min }\right)$. Full width at half maximum (FWHM, c) is normalized in respect to the width of the first recorded profile (compare with Figure 5 in the main text). Signal-to-noise ratio (SNR, d) was calculated for singly-stamped lines vs. non- 
stamped background. Note that for PMMA, in the last two points (132 and $155 \mathrm{~h}$ ), establishing the proper value of FWHM was impossible due to very low SNR and contrast. Every point was calculated from four separate profiles.

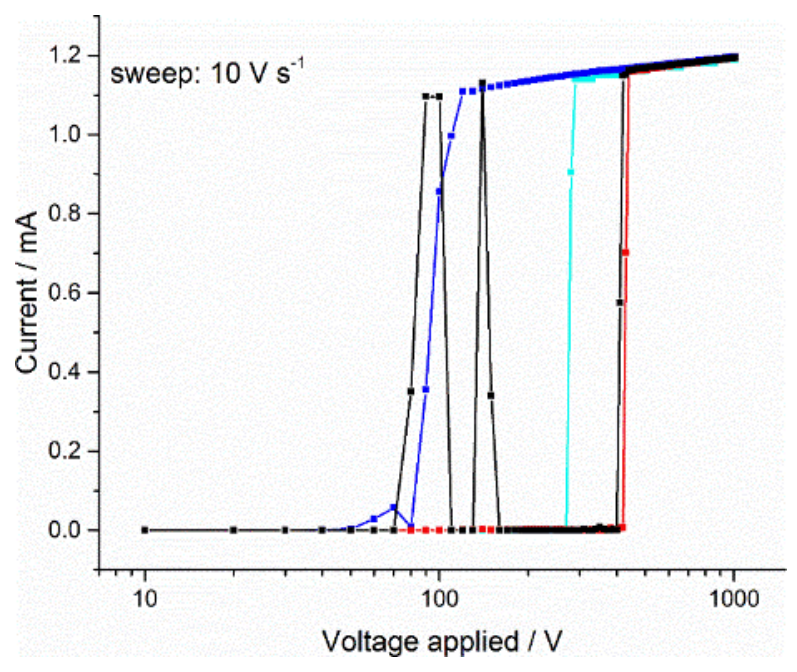

Figure S8. Dielectric breakdown of a 1- $\mu$ m-thick PMMA film deposited on p-type, B doped Si contact electrified with a PDMS stamp $\left(1 \mathrm{~cm}^{2}\right.$ area and presenting an array of 5 - $\mu$ m-thick lines separated by $9 \mu \mathrm{m}$ ) metalized with $100 \mathrm{~nm} \mathrm{Au} / \mathrm{Pd}$. The bias (sweep from 0 to $1000 \mathrm{~V}, 10 \mathrm{~V} / \mathrm{s}$ ) was applied between the stamp and the bottom of the Si substrate. Graph presents results obtained for four separate samples. 

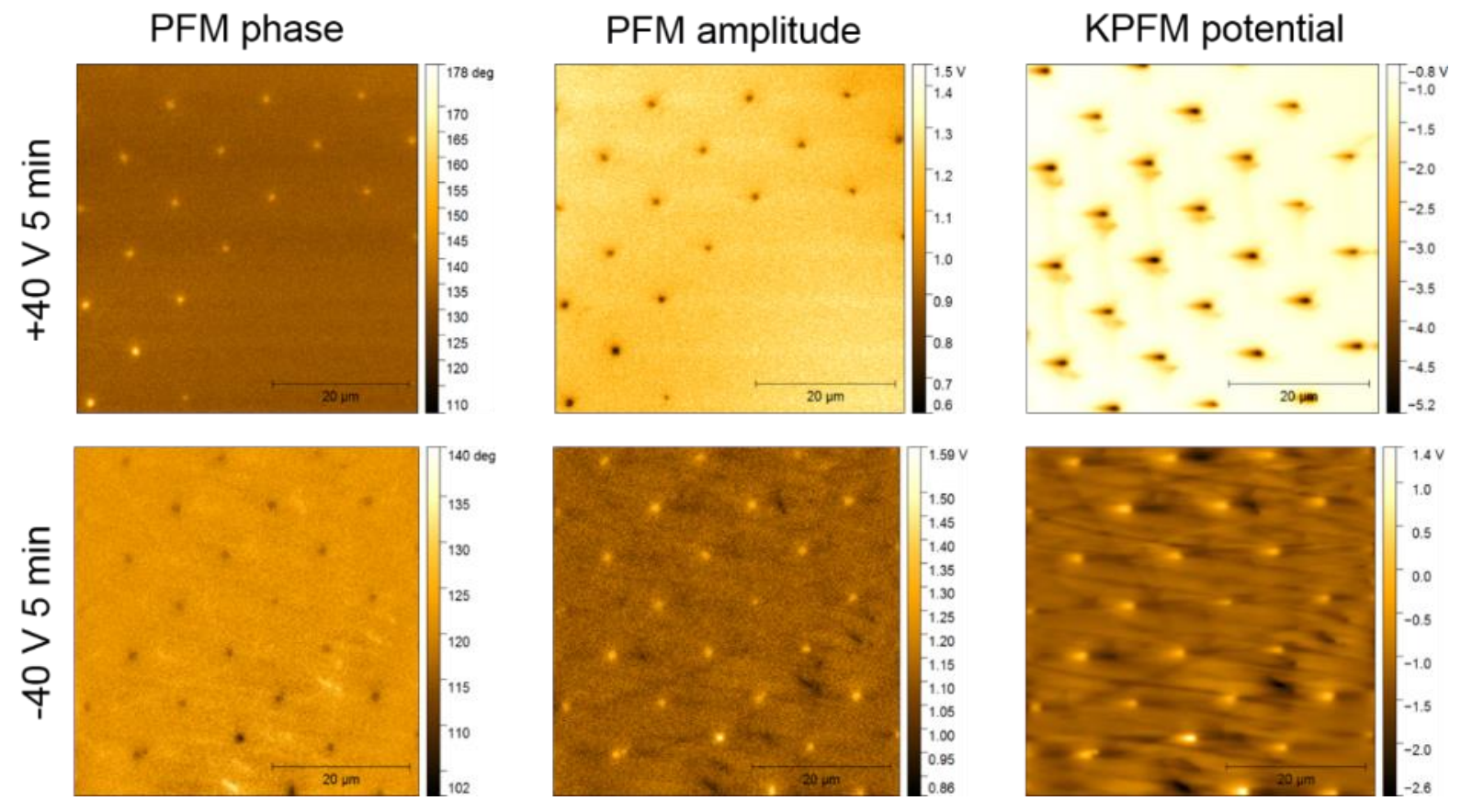

Figure S9. PFM (phase and amplitude) and KPFM (potential) images of samples treated with $+40 \mathrm{~V}$ (top row) or $-40 \mathrm{~V}$ (bottom row) electrical bias applied to the Au/Pd-coated PDMS stamp for $5 \mathrm{~min}$. Dot-shaped features were chosen for clarity of presentation. Images were leveled by mean-plane subtraction using Gwyddion software. 

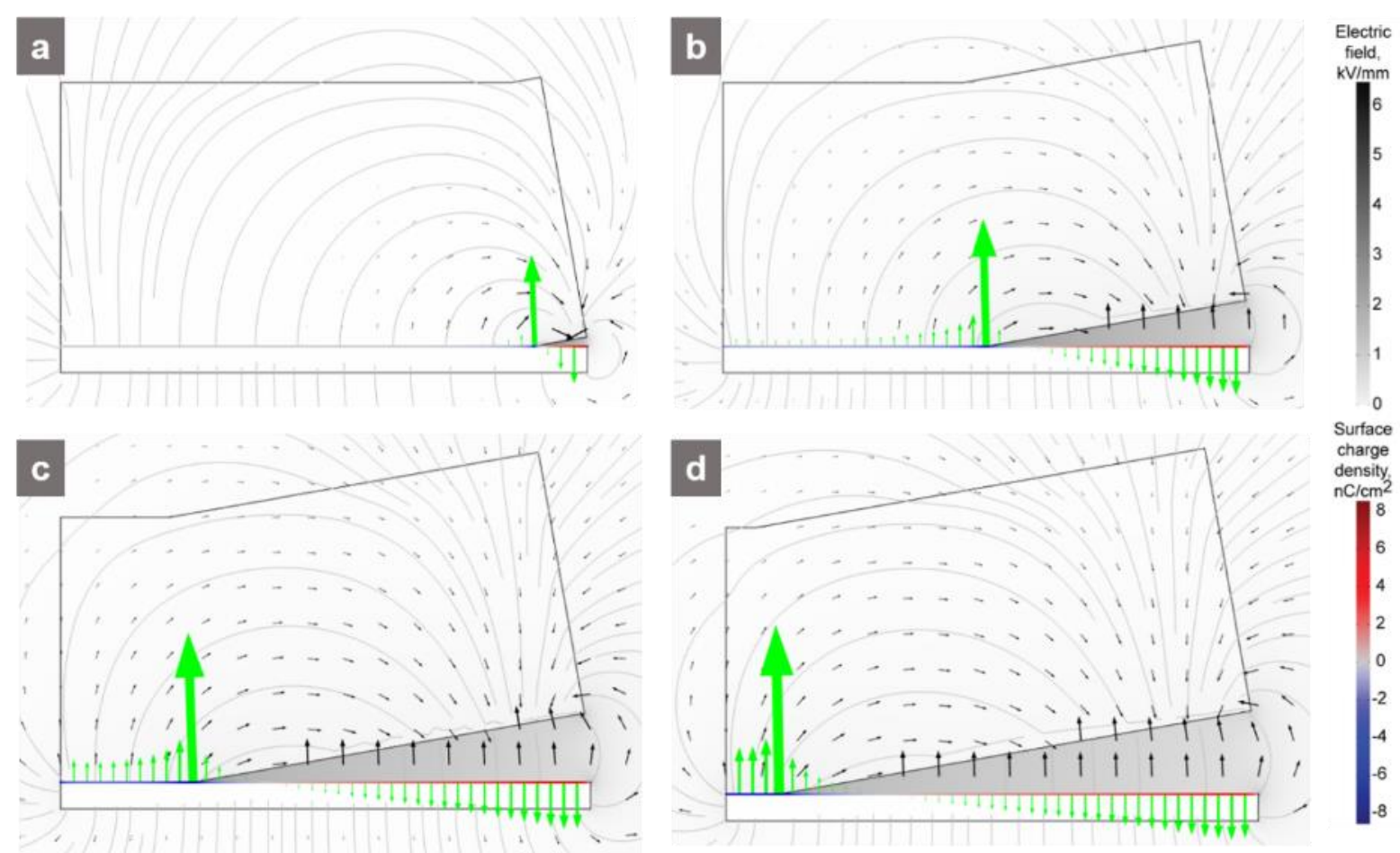

Figure S10. Development of electric fields created during delamination of the PDMS stamp from the PMMA film supported on a conductive substrate. Gray scale corresponds to the magnitude of the field, lines and arrows show directionality of the field. Green arrows depict changes in the electric field in the middle of PMMA film. Color scale corresponds to surface charge density generated at the PMMA/air interface. 

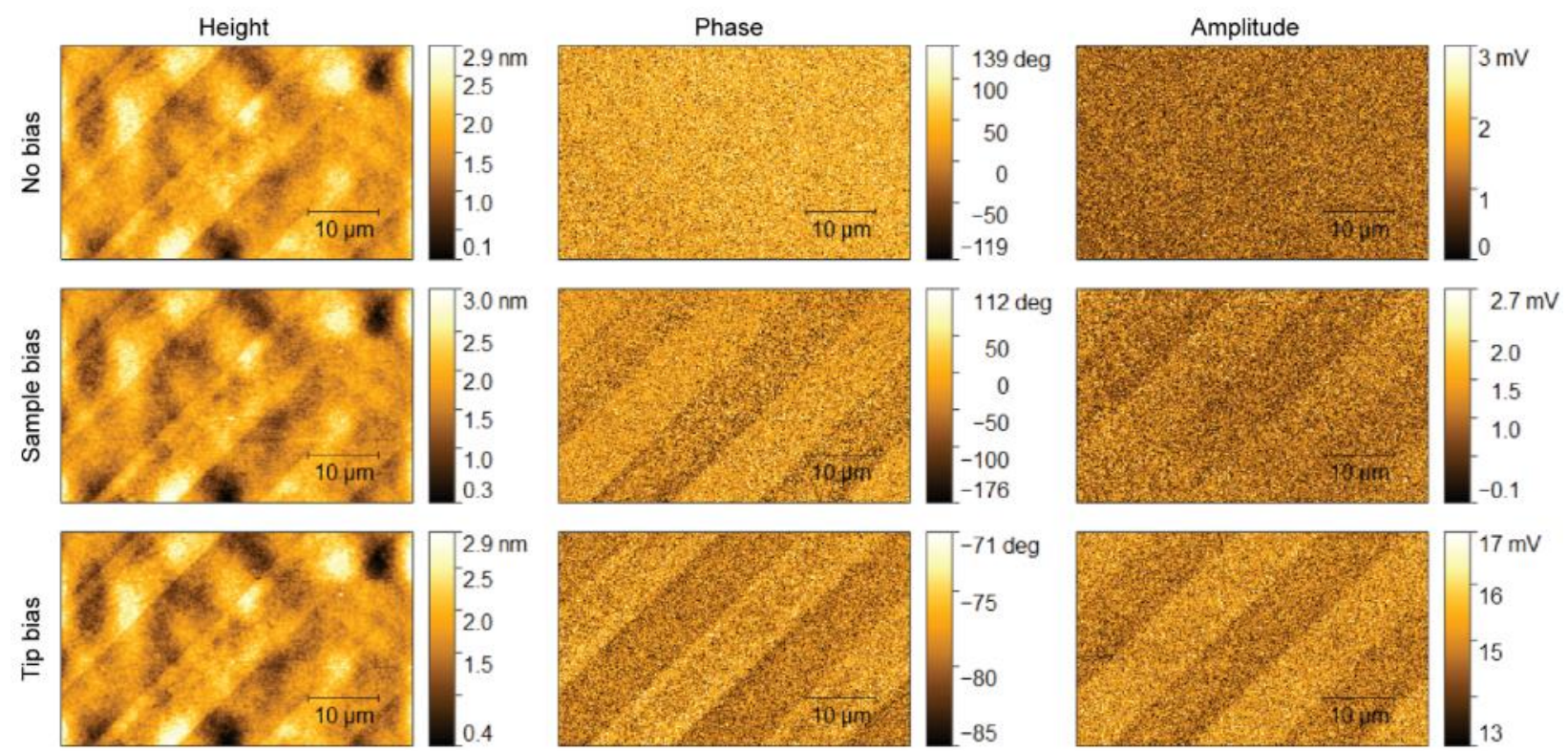

Figure S11. PFM of the PMMA film stamped with PDMS stamp featured with an array of parallel, 8- $\mu$ m-thick lines. PFM readouts obtained with different applied biases - no bias (top row), $10 \mathrm{~V}$ AC bias applied to the sample (middle row), $10 \mathrm{~V}$ AC bias applied to the tip (bottom row). As seen, height (left column, background-corrected) is not affected by the tip bias, while phase (middle column) and amplitude (right column) are only visible when the bias is applied. Moreover, the direction of the phase changes when the bias direction is changed which confirms that the PMMA film is polarized and not only under electrostriction (in such a case, the stripes would be visible also without the applied bias, and the direction of the phase would not change with the change of direction of the bias). For clarity of presentation, images were leveled by mean-plain subtraction using Gwyddion software. 


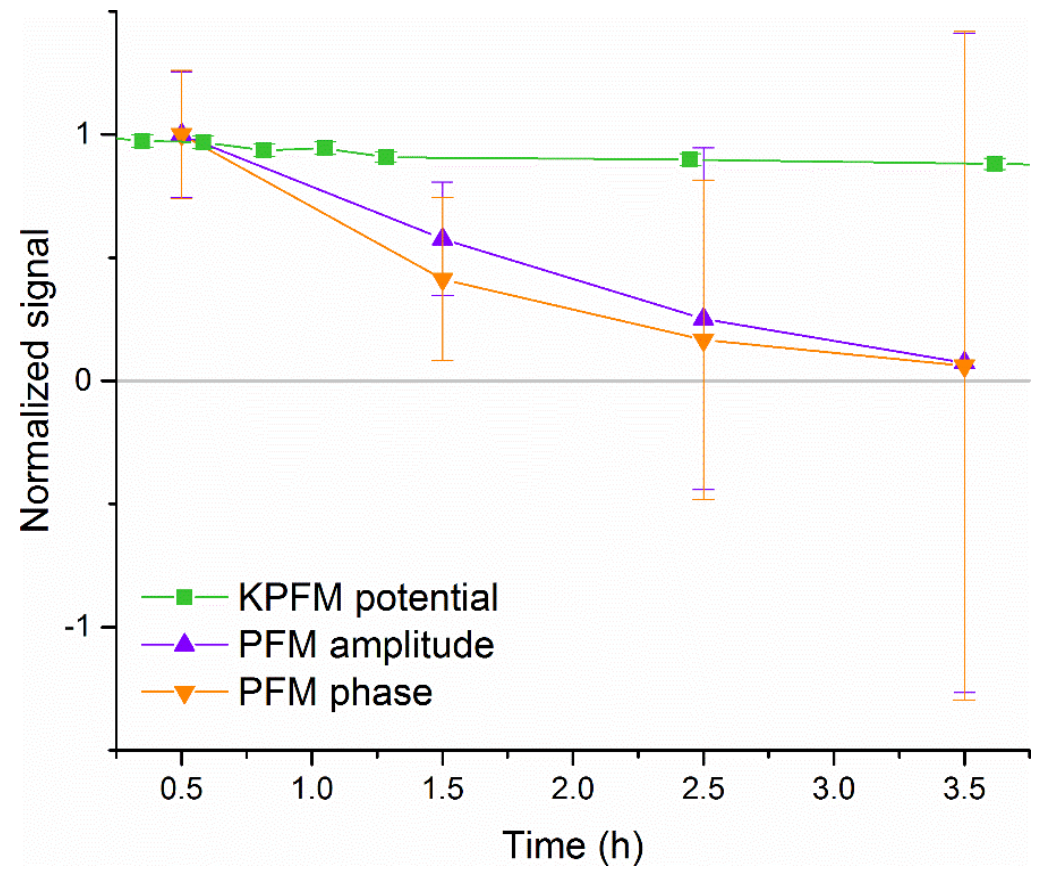

Figure S12. Comparison of normalized decay profiles obtained for potential readout of KPFM (depicted by full green squares, same data as in Figure 5a) and amplitude (violet triangles) and phase (orange triangles) readouts of PFM obtained from two identical samples (1- $\mu$ m-thick PMMA on p-type, B doped Si stamped for 45 min with 5:1 PDMS stamp featured with $7 \mu \mathrm{m}$ lines; one sample used for KPFM measurement, the other for PFM measurement). Note that PFM works in contact mode with applied bias thus changing the initial parameters of the tested sample with every scan, while KPFM is a non-destructive method. Data shows mean \pm SD for at least four profiles. 


\section{Supplementary references:}

(S1) Kang, S. J.; Park, Y. J.; Bae, I.; Kim, K. J.; Kim, H. C.; Bauer, S.; Thomas, E. L.; Park, C. Printable Ferroelectric PVDF/PMMA Blend Films with Ultralow Roughness for Low Voltage Non-Volatile Polymer Memory. Adv. Funct. Mater. 2009, 19 (17), 2812-2818.

(S2) Inaba, H.; Ohmi, T.; Yoshida, T.; Okada, T. Neutralization of Static Electricity by Soft XRays and Vacuum UV Radiation. J. Electrostat. 1994, 33 (1), 15-42.

(S3) Baytekin, H. T.; Baytekin, B.; Soh, S.; Grzybowski, B. A. Is Water Necessary for Contact Electrification? Angew. Chemie - Int. Ed. 2011, 50 (30), 6766-6770.

(S4) Poulsen, M.; Ducharme, S. Why Ferroelectric Polyvinylidene Fluoride Is Special. IEEE Trans. Dielectr. Electr. Insul. 2010, 17 (4), 1028-1035.

(S5) Gross, B.; De Moraes, R. J. Polarization of the Electret. J. Chem. Phys. 1962, 37 (4), 710 713.

(S6) Qian, K.; Nguyen, V. C.; Chen, T.; Lee, P. S. Novel Concepts in Functional Resistive Switching Memories. J. Mater. Chem. C 2016, 4 (41), 9637-9645.

(S7) Małecki, J. A. Linear Decay of Charge in Electrets. Phys. Rev. B - Condens. Matter Mater. Phys. 1999, 59 (15), 9954-9960.

(S8) Cuniot-Ponsard, M. Kelvin Probe Force Microscopy and Electrostatic Force Microscopy Responses to the Polarization in a Ferroelectric Thin Film: Theoretical and Experimental Investigations. J. Appl. Phys. 2013, 114 (1), 014302.

(S9) Jesse, S.; Baddorf, A. P.; Kalinin, S. V. Dynamic Behaviour in Piezoresponse Force Microscopy. Nanotechnology 2006, 17 (6), 1615-1628.

(S10) Elias, G.; Glatzel, T.; Meyer, E.; Schwarzman, A.; Boag, A.; Rosenwaks, Y. The Role of the Cantilever in Kelvin Probe Force: Microscopy Measurements. Beilstein J. Nanotechnol. 2011, 2 (1), 252-260.

(S11) Vasudevan, R. K.; Balke, N.; Maksymovych, P.; Jesse, S.; Kalinin, S. V. Ferroelectric or Non-Ferroelectric: Why so Many Materials Exhibit "Ferroelectricity" on the Nanoscale. 
Appl. Phys. Rev. 2017, 4 (2), 021302.

(S12) Seol, D.; Kim, B.; Kim, Y. Non-Piezoelectric Effects in Piezoresponse Force Microscopy. Curr. Appl. Phys. 2017, 17 (5), 661-674.

(S13) Das, S.; Wang, B.; Cao, Y.; Rae Cho, M.; Jae Shin, Y.; Mo Yang, S.; Wang, L.; Kim, M.; Kalinin, S. V.; Chen, L.-Q.; et al. Controlled Manipulation of Oxygen Vacancies Using Nanoscale Flexoelectricity. Nat. Commun. 2017, 8 (1), 615.

(S14) Glinchuk, M. D.; Eliseev, E. A.; Li, G.; Zeng, J.; Kalinin, S. V.; Morozovska, A. N. Ferroelectricity Induced by Oxygen Vacancies in Relaxors with Perovskite Structure. Phys. Rev. B 2018, 98 (9), 094102.

(S15) Christman, J. A.; Woolcott, R. R.; Kingon, A. I.; Nemanich, R. J. Piezoelectric Measurements with Atomic Force Microscopy. Appl. Phys. Lett. 1998, 73 (26), 38513853.

(S16) Jungk, T.; Hoffmann, Á.; Soergel, E. Quantitative Analysis of Ferroelectric Domain Imaging with Piezoresponse Force Microscopy. Appl. Phys. Lett. 2006, 89 (16), 163507.

(S17) Gomez, A.; Puig, T.; Obradors, X. Diminish Electrostatic in Piezoresponse Force Microscopy through Longer or Ultra-Stiff Tips. Appl. Surf. Sci. 2018, 439, 577-582.

(S18) Kalinin, S. V.; Bonnell, D. A. Local Potential and Polarization Screening on Ferroelectric Surfaces. Phys. Rev. B - Condens. Matter Mater. Phys. 2001, 63 (12), 1-13.

(S19) Kalinin, S. V.; Bonnell, D. A. Contrast Mechanism Maps for Piezoresponse Force Microscopy. J. Mater. Res. 2002, 17 (5), 936-939.

(S20) Kalinin, S. V.; Gruverman, A.; Rodriguez, B. J.; Shin, J.; Baddorf, A. P.; Karapetian, E.; Kachanov, M. Nanoelectromechanics of Polarization Switching in Piezoresponse Force Microscopy. J. Appl. Phys. 2005, 97 (7), 1-6.

(S21) Kalinin, S. V.; Rodriguez, B. J.; Jesse, S.; Karapetian, E.; Mirman, B.; Eliseev, E. A.; Morozovska, A. N. Nanoscale Electromechanics of Ferroelectric and Biological Systems: A New Dimension in Scanning Probe Microscopy. Annu. Rev. Mater. Res. 2007, 37 (1), 189-238. 
(S22) Denning, D.; Alilat, S.; Habelitz, S.; Fertala, A.; Rodriguez, B. J. Visualizing Molecular Polar Order in Tissues via Electromechanical Coupling. J. Struct. Biol. 2012, 180 (3), 409-419.

(S23) Deng, Q.; Liu, L.; Sharma, P. Electrets in Soft Materials: Nonlinearity, Size Effects, and Giant Electromechanical Coupling. Phys. Rev. E - Stat. Nonlinear, Soft Matter Phys. 2014, $90(1), 1-7$.

(S24) Franke, K. Evaluation of Electrically Polar Substances by Electric Scanning Force Microscopy. Part II: Measurement Signals Due to Electromechanical Effects. Ferroelectr. Lett. Sect. 1995, $19(1-2), 35-43$.

(S25) Franke, K.; Huelz, H.; Weihnacht, M. How to Extract Spontaneous Polarization Information from Experimental Data in Electric Force Microscopy. Surf. Sci. 1998, 415 (1-2), 178-182.

(S26) Dipole Moment «Rosamonte’s Physical Chemistry Website https://physicalchemistryrosamonte.wordpress.com/material-balances/material-balanceson-a-crystallizer/physical-properties-of-pure-methanol/dipole-moment/ (accessed Jul 31, 2019).

(S27) Vinyl acetate http://www.microkat.gr/msdspd90-99/Vinyl acetate.html (accessed Jul 31, 2019).

(S28) Shinyashiki, N.; Miyara, M.; Nakano, S.; Yamamoto, W.; Ueshima, M.; Imoto, D.; Sasaki, K.; Kita, R.; Yagihara, S. Dielectric Relaxation Strength and Magnitude of Dipole Moment of Poly(Vinyl Pyrrolidone)in Polar Solutions. J. Mol. Liq. 2013, 181, 110-114.

(S29) Kivelson, D.; Wilson, E. B.; Lide, D. R. Microwave Spectrum, Structure, Dipole Moment, and Nuclear Quadrupole Effects in Vinyl Chloride. J. Chem. Phys. 1960, 32 (1), 205-209.

(S30) Yaws, C. L. Thermophysical Properties of Chemicals and Hydrocarbons: Second Edition; Elsevier, 2014.

(S31) Brandrup, J.; Immergut, E. H.; Grulke, E. A. Polymer Handbook; Wiley-Interscience, New York, 2003. 
(S32) Tonelli, A. E. Conformational Characteristics of Poly(N-Vinyl Pyrrolidone). Polymer. 1982, 23 (5), 676-680.

(S33) Matsui, H.; Mishchenko, A. S.; Hasegawa, T. Carrier Localization in Organic Transistors with Polymer Gate Insulators. In Extended Abstracts of the 2013 International Conference on Solid State Devices and Materials; The Japan Society of Applied Physics, 2013.

(S34) Jenkins, A. D. Polymer Science: A Materials Science Handbook; North-Holland Publishing Company: Amsterdam, London, 1972.

(S35) Kronjaeger, J. Electrical properties of insulators http://www.kronjaeger.com/hvold/hv/tbl/prop.html (accessed Sep 19, 2019).

(S36) Pinar, F. Fourier Transform Infrared Spectroscopy of Organic Dielectric / Organic Semiconductor Interface, Johannes Kepler Universität, 2007.

(S37) Roff, W. J.; Scott, J. R. Handbook of Common Polymers Fibres, Films, Plastics and Rubbers; Butterworths, 1971.

(S38) Rawat, A.; Mahavar, H. K.; Tanwar, A.; Singh, P. J. Study of Electrical Properties of Polyvinylpyrrolidone/Polyacrylamide Blend Thin Films, Bull. Mater. Sci. 2014, 37, 273 279. 